\title{
ALTERAÇÕES FISIOLÓGICAS DA MORTE ENCEFÁLICA EM POTENCIAIS DOADORES DE ÓRGÃOS E TECIDOS PARA TRANSPLANTES
}

\author{
Physiological changes of brain death in potential donors of organs and tissues for \\ transplantation \\ Los cambios fisiológicos de la muerte cerebral en potenciales donadores de órganos y \\ tej idos para trasplante
}

Sarah Gabriel Freire $\quad$ Izaura Luzia Silvério Freire ${ }^{2}$

Quinidia Lúcia Duarte de Almeida Quithé de Vasconcelos ${ }^{4}$

Juliana Teixeira Jales Menescal Pinto ${ }^{3}$

Gilson De Vasconcelos Torres ${ }^{5}$

\section{RESUMO}

Objetivou-se descrever as alterações fisiológicas da morte encefálica em potenciais doadores de órgãos e tecidos para transplantes. Estudo exploratório descritivo com dados prospectivos e abordagem quantitativa realizado nas unidades de emergência e terapia intensiva adulto de um hospital de Pernambuco no período de abril a outubro de 2011. A população constou de 32 potenciais doadores de órgãos e tecidos para transplantes. Após aprovação do Comitê de Ética em Pesquisa, os dados foram coletados, tabulados e analisados pela estatística descritiva pelo software SPSS 15.0 e apresentados em forma de tabelas. As alterações fisiológicas foram: hipotensão arterial (100\%), hipotermia $(75,0 \%)$, hipernatremia $(62,5 \%)$, diabetes insipidus (37,5\%), hiperglicemia (32,3\%), infecção $(25,0 \%)$, hipertensão arterial $(9,4 \%)$ e úlcera de córnea $(3,1 \%)$. Acredita-se que o conhecimento dessas alterações possibilita à equipe de saúde direcionar o cuidado ao potencial doador segundo as suas necessidades e, assim, manter o órgão/tecido viável para transplante.

Palavras-chave: Enfermagem. Obtenção de tecidos e órgãos. Morte encefálica.

\begin{abstract}
The objective was to describe the physiologic changes of brain death in potential donors of organs and tissues for transplantation. Exploratory descriptive study with prospective data and quantitative approach carried out in emergency and intensive care units hospital adult, in the period from April to October 2011. The population consisted of 32 potential donors of organs and tissues for transplantation. After approval of Ethics Committee, data were collected, tabulated and analyzed by descriptive statistics by SPSS 15.0 software and presented in tables. Physiological changes were: hypotension (100\%), hypothermia (75\%), hypernatremia $(62,5 \%)$, diabetes insipidus $(37,5 \%)$, hyperglycemia $(32,3 \%)$, infection $(25,0 \%)$, hypertension $(9,4 \%)$ and corneal ulcer $(3,1 \%)$. It was found that knowledge of these changes allows the team of health care to direct the potential donors according to their needs and thus keep the organ/tissue viable for transplant.
\end{abstract}

Keywords: Nursing. Tissue and Organ Procurement. Brain Death.

\section{Resumen}

El objetivo del estudio fue describir los cambios fisiológicos de la muerte cerebral en los potenciales donadores de órganos y tejidos para trasplante. Investigación exploratoria, descriptiva, con datos prospectivos y enfoque cuantitativo, hecho en las unidades de emergencia y de cuidados intensivos de adultos de un hospital de Pernambuco en el periodo de abril a octubre de 2011. La población fue formada por 32 potenciales donadores de órganos y tejidos para trasplante. Después de la aprobación en el Comité Ético de Investigación, los datos han sido recogidos, tabulados y analizados mediante estadística descriptiva por el programa informático SPSS 15.0, siendo presentados en forma de tablas. Los cambios fisiológicos fueron: hipotensión (100\%), hipotermia $(75,0 \%)$, hipernatremia $(62,5 \%)$, diabetes insípida $(37,5 \%)$, infección $(25,0 \%)$, hipertensión arterial $(9,4 \%)$ y la úlcera de córnea $(3,1 \%)$. Se cree que el conocimiento de estos cambios permite al equipo de atención de la salud dirigir sus acciones al potencial donador de acuerdo a sus necesidades y así mantener los órganos/tejidos viables para el trasplante.

Palabras-clave: Enfermería. Obtención de Tejidos y Órganos. Muerte Encefálica.

\footnotetext{
${ }^{1}$ Enfermeira. Especialista em terapia intensiva pelo Hospital da Restauração/Recife. Natal- RN. Brasil. Email: sarahgabrielfreire@gmail.com; ${ }^{2}$ Enfermeira. Professora Mestre da Escola de Enfermagem da Universidade Federal do Rio Grande do Norte/UFRN. Doutoranda do Programa de Pós-Graduação em Enfermagem/UFRN. Membro do Grupo de Pesquisa Incubadora de Procedimentos de Enfermagem/UFRN. Natal-RN. Brasil. Email: izaurafreire@hotmail.com; ${ }^{3}$ Enfermeira. Professora Mestre da Escola de Enfermagem da Universidade Federal do Rio Grande do Norte/UFRN. Membro do Grupo de Pesquisa Saúde e Sociedade da Escola de Enfermagem/UFRN. Natal-RN. Brasil. Email: jujales@hotmail.com; ${ }^{4}$ Acadêmica de Enfermagem da Universidade Federal do Rio Grande do Norte/ UFRN. Membro do Grupo de Pesquisa Incubadora de Procedimentos de Enfermagem/UFRN. Natal-RN. Brasil. Email: quinidia@hotmail.com; ${ }^{5}$ Enfermeiro. Professor Pós-Doutor do Departamento de Enfermagem e do Programa de Pós-Graduação em Enfermagem da Universidade Federal do Rio Grande do Norte/UFRN. Coordenador do Grupo de Pesquisa Incubadora de Procedimentos de Enfermagem/UFRN. Natal-RN. Brasil. Email: gilsonvtorres@hotmail.com.
} 


\section{INTRODUÇÃO}

Por muito tempo, o conceito de morte esteve atrelado à ausência de batimentos cardíacos ou movimentos respiratórios espontâneos. Com o passar do tempo esse conceito se modificou, uma vez que, atualmente, se torna possível manter as funções vitais do ser humano, por longos períodos, mesmo sem o funcionamento do encéfalo ${ }^{1}$.

Historicamente, a primeira concepção de Morte Encefálica (ME) foi desenvolvida em 1959, por um grupo de neurologistas franceses, pela condição clínica em que se encontra um encéfalo mor to em um corpo vivo, denominada na época de coma dépassé?.

Após anos de discussões sobre esse tema, foi criada, em 1981, nos Estados Unidos da América (EUA), uma comissão presidencial que definiu a morte como a cessação irreversível das funções circulatória e respiratória ou a parada irreversível do funcionamento de todo o encéfalo. Esses critérios são utilizados atualmente para se realizar o diagnóstico da ME?2.

No Brasil, esses critérios foram legalmente adotados em 1997, por meio da Resolução $n^{0} 1.480 / 97$ do Conselho Federal de Medicina (CFM), a qual define a ME como "a parada total e irreversível das funções encefálicas, de causa conhecida e constatada de modo indiscutível", constituindo morte para efeitos clínico, legal e/ou social'.

Dessa forma, a evolução do conceito de ME foi fundamental para viabilidade da doação de órgãos, uma vez que, diagnosticada mediante critérios legais e pré-definidos, segue-se a manutenção das funções vitais para efetivar o transplante ${ }^{3}$.

É importante ressaltar que a causa da morte deve ser conhecida e bem definida e que o diagnóstico de ME deve ser compulsoriamente notificado para a Central de Notificação, Captação e Doação de Órgãos para transplante (CNCDO) de cada estado!'

Para potencializar o número de transplantes com o doador falecido, é necessário garantir uma adequada preservação e posterior viabilidade desses órgãos até a extração. Isso implica investimentos em materiais e equipamentos especializados, equipe de profissionais capacitados que saibam identificar e diagnosticar precocemente a ME, e, principalmente, reconhecer as alterações fisiológicas apresentadas por esse paciente 4 .

A ME ocasiona efeitos deletérios sobre os Potenciais Doadores (PDs), tendo como principais alterações fisiológicas decorrentes desse evento a hipotensão arterial, prevalente em $81 \%$ dos casos, seguida do diabetes insipidus (DI), que surge em $78 \%$, e coagulação intravascular disseminada, presente em $28 \%$ dos PDs 5 .

Essas alterações, quando não tratadas adequadamente, são as principais causas da não efetivação do transplante de órgãos e tecidos. Para evitar esse problema, torna-se necessário o conhecimento dos profissionais intensivistas quanto ao reconhecimento precoce e à consequente manutenção deste organismo a fim de preservar os órgãos antes que ocorra a parada cardíaca. Em 2011, a parada cardíaca surgiu como a segunda causa de não efetivação da doação de órgãos no país, responsável por $17 \%$ dos casos $^{6-7}$.

Apesar de o Brasil ser o país da América Latina com o maior número de doadores com órgãos transplantados, com um total de 2.019 transplantes no ano de 2011, ainda há 27.827 pessoas na fila de espera para transplante. Nesse mesmo ano, foram notificados 7.238 PDs e somente $10,6 \%$ destes chegaram a efetivar a doação, ou seja, apenas a metade dos PDs que se estima existir são notificados, e somente um, em cada quatro, tem os órgãos transplantados ${ }^{7}$.

Esses dados não diferem do Estado de Pernambuco, onde, apesar de ter obtido uma colocação de destaque, o $10^{\circ}$ lugar em relação aos 24 estados brasileiros que realizam transplantes, foram notificados, em 2011, 359 PDs e, destes, apenas $67(18,7 \%)$ tiveram seus órgãos transplantados. Dentre as principais causas da não efetivação da doação nesse estado estão a não autorização familiar (54\%), a contraindicação médica (17\%), a ME não confirmada (28\%) e a parada cardíaca $(8 \%)^{7}$.

Ressalta-se, assim, que, para otimizar esses dados, é necessário que a equipe de saúde que assiste o PD tenha conhecimento das alterações fisiológicas decorrentes do processo de ME, pois elas se constituem em um processo complexo que muda a fisiologia de todos os sistemas orgânicos, conduzindo à disfunção múltipla de órgãos, repercutindo na quantidade e qualidade dos órgãos a serem transplantados.

Diante do exposto e a partir da vivência dos autores em Unidades de Terapia Intensiva (UTIS) de hospitais de referência para atendimentos de urgência e emergência, surgiu o interesse por este campo de estudo. Nessas unidades, a maior parte das admissões tem como diagnóstico médico as doenças cerebrovasculares e as causas externas (acidentes e violência). Esses pacientes, comumente em estado grave, evoluem para ME, tornando-se PDs, mas nem sempre conseguem ter seus órgãos disponibilizados para transplante.

Paralelo a essa realidade, alguns autores afirmam que uma das causas para a não efetivação do transplante de órgãos e tecidos deve-se ao conhecimento insuficiente dos profissionais de saúde sobre a fisiologia e fisiopatologia da ME; e consideram como aspecto fundamental no cuidado ao PD a assistência sistematizada respaldada na avaliação clínica, no diagnóstico precoce de ME, no reconhecimento das alterações fisiológicas decorrentes desse processo, no planejamento do tratamento adequado, na implementação dos cuidados, na evolução e na reavaliação das condutas e tratamento, além de trabalho educativo permanente ${ }^{3-5}$.

Diante do exposto e preocupados com o baixo índice de efetivação de transplante, resolvemos fazer este estudo com o objetivo de descrever as alterações fisiológicas da ME em PDs de órgãos e tecidos para transplantes. 


\section{METODOLOGIA}

Trata-se de um estudo exploratório descritivo com dados prospectivos e abordagem quantitativa, realizado nas unidades de emergência e terapia intensiva adulto do Hospital da Restauração (HR), em Recife/ PE, no período de abril a outubro de 2011.

A população do estudo foi constituída por 32 PDs de órgãos e tecidos para transplantes, internados nas unidades de emergência e terapia intensiva adulto do citado hospital. Excluíram-se do estudo indivíduos com diagnóstico de ME, com contraindicações médicas para a doação, e indivíduos com pontuação de três na Escala de Coma de Glasgow, sem causa de coma definida por meio de exames clínicos e laboratoriais.

0 instrumento de coleta de dados utilizado foi elaborado pelos próprios pesquisadores, tomando como base os fundamentos preconizados pela literatura científica sobre 0 assunto ${ }^{1,3-5,8-9}$. Trata-se de um roteiro estruturado do tipo checklist, que descreve as variáveis a serem pesquisadas à esquerda e as respostas de cada uma delas com "presente" ou "ausente" à direita.

Este instrumento foi dividido em duas partes: a primeira se refere à caracterização do $\mathrm{PD}$, com variáveis sobre sexo, idade, procedência, setor de internação, diagnóstico médico, resultado da doação e causa da não efetivação da doação. A segunda parte do instrumento foi composta pelas variáveis referentes às alterações fisiológicas decorrentes da ME, como a hipertensão arterial, hipotensão arterial, hipotermia, infecções, úlceras de córnea e os distúrbios metabólicos como o diabetes insipidus, alteraç̃̃es no sódio, no potássio e na glicose.

Para efeito deste estudo, os seguintes conceitos foram considerados segundo a definição da literatura científica ${ }^{1,3-5,7-9}$ :

Hipertensão arterial: pressão arterial (PA) sistólica superior a $140 \mathrm{mmHg}$ e diastólica superior a $90 \mathrm{mmHg}$.

Hipotensão arterial: PA sistólica inferior a 90 $\mathrm{mmHg}$ ou PA média inferior a $65 \mathrm{mmHg}$.

- Hipotermia: temperatura inferior a $35^{\circ} \mathrm{C}$.

- Infecções: diagnosticada por meio de culturas positivas de material biológico e alterações nas radiografias de tórax, descritas no prontuário.

- Úlceras de córnea: lesão de córnea diagnosticada e registrada no prontuário pelo médico.

Diabetes insipidus: debito urinário acima de $4 \mathrm{ml} /$ $\mathrm{kg} / \mathrm{h}$.

Alterações nos eletrólitos: sódio sérico inferior a $130 \mathrm{mEq} / \mathrm{L}$ ou superior a $150 \mathrm{mEq} / \mathrm{L}$ e potássio sérico inferior a 3,5 mEq/L ou superior a 5,5 $\mathrm{mEq} / \mathrm{L}$.

- Alterações na glicose: glicemia inferior a 100 mg/ dl ou superior a $200 \mathrm{mg} / \mathrm{dl}$.

A coleta de dados foi realizada pelos pesquisadores após a realização de um treinamento e a pré-testagem do instrumento com $10 \%$ da amostra, ou seja, 3 PDs. Ao iniciar a pesquisa, foi solicitado o consentimento do cônjuge ou parente até segundo grau do $\mathrm{PD}^{9}$ em participar do estudo, mediante a assinatura do termo de consentimento livre e esclarecido. As informações foram coletadas diariamente pela técnica de observação sistemática não participante, de documentos institucionais como livros de ocorrências e prontuários.

Em seguida, os dados foram tabulados e analisados com uso da estatística descritiva e apresentados em forma de tabelas, com frequência relativa e absoluta. Para tanto, utilizou-se o software SPSS 15.0.

Foram obedecidos todos os princípios éticos e legais que regem a pesquisa em seres humanos, preconizados na Resolução do Conselho Nacional de Saúde n. 196/96, manifestado pela aprovação do protocolo do registro do Comitê de Ética em Pesquisa do HR n. 0026.0.102.000-11.

\section{RESULTADOS}

No primeiro momento serão apresentados os dados de caracterização dos 32 PDs, e em um segundo momento serão abordadas as alterações fisiológicas decorrentes da ME apresentadas pelos PDs de órgãos e tecidos para transplantes.

\section{Caracterização da amostra estudada}

A maior parte dos PDs era do sexo masculino $(65,6 \%)$, com faixa etária predominante de 21 a 30 anos $(28,1 \%)$ e média de idades de 35 anos. A idade mínima foi 14 e a máxima, 72 anos. A maioria era procedente da região metropolitana de Recife $(56,3 \%)$, internados no setor de emergência (59,4\%), com diagnóstico médico de TCE $(43,7 \%)$. Quanto aos resultados das doações, observa-se que a maioria dos PDs foram não doadores $(65,6 \%)$. A principal causa de não efetivação da doação foi a parada cardíaca $(61,9 \%)$ seguida da recusa familiar $(38,1 \%)$.

\section{Alterações fisiológicas da morte encefálica em potenciais doadores de órgãos e tecidos para transplantes.}

Percebe-se que a alteração fisiológica mais prevalente foi a hipotensão arterial, presente em $100 \%$ dos PDs, seguida da hipotermia (75\%), hipernatremia (62,5\%), DI (37,5\%), hiperglicemia (32,3\%), infecção $(25,0 \%)$ e úlceras de córnea $(3,1 \%)$. A hipertensão arterial $(9,4 \%)$ estava presente antes da abertura do protocolo de ME. 
Tabela 1. Caracterização sociodemográfico dos potenciais de órgãos e tecidos para transplante. HR - Pernambuco/PE, 2012

\begin{tabular}{llcc}
\hline \multicolumn{2}{c}{ CARACTERISTICAS SOCIODEMOGRÁFICAS } & $\mathrm{n}$ & $\%$ \\
\hline Sexo & Masculino & 21 & 65,6 \\
\multirow{2}{*}{ Faixa etária } & Feminino & 11 & 34,4 \\
& Até 20 anos & 7 & 21,9 \\
& $21-30$ anos & 9 & 28,1 \\
& $31-40$ anos & 4 & 12,5 \\
& $41-50$ anos & 7 & 21,9 \\
& $51-60$ & 2 & 6,3 \\
\multirow{2}{*}{ Procedência } & Acima de 60 anos & 3 & 9,4 \\
\multirow{2}{*}{ Setor de internação } & Região metropolitana & 18 & 56,3 \\
& Interior & 14 & 43,8 \\
Diagnóstico médico & Emergência & 19 & 59,4 \\
& UTI & 13 & 40,6 \\
& TCE & 14 & 43,7 \\
Resultado da doação & AVC & 11 & 34,4 \\
& Outras causas & 7 & 21,9 \\
Causas da não doação & Não doador & 21 & 65,6 \\
& Doador & 11 & 34,4 \\
& Parada cardíaca & 13 & 61,9 \\
\hline *Fonte: própria da pesquisa & Recusa familiar & 8 & 38,1 \\
\hline
\end{tabular}

Tabela 2. Alteraç̧ões fisiológicas apresentadas pelos PDs de órgãos e tecidos para transplante. HR - Pernambuco/PE, 2012.

\begin{tabular}{lcc}
\hline \multicolumn{1}{c}{ ALTERAÇÕES FISIOLÓGICAS } & $\mathbf{n}$ & $\mathbf{\%}$ \\
\hline Hipotensão arterial & 32 & 100,0 \\
Hipertensão arterial & 03 & 9,4 \\
Hipotermia & 24 & 75,0 \\
Hipernatremia & 20 & 62,5 \\
Diabetes insipidus & 12 & 37,5 \\
Hiperglicemia & 10 & 32,3 \\
Infecção & 08 & 25,0 \\
Úlcera de córnea & 01 & 3,1 \\
\hline
\end{tabular}

*Fonte: própria da pesauisa

\section{DISCUSSÃO}

No presente estudo observou uma predominância do sexo masculino. Esse fato é justificado pela prevalência dos homens em traumas violentos graves, como ferimentos por arma de fogo ou branca, ou ainda por esse sexo ser o mais envolvido em acidentes de trânsito graves ${ }^{8,11}$.

Com relação à distribuição por idade, constatamos que a amplitude etária variou de 14 a 72 anos, situando-se a média de idades nos 35 anos. Assim, consideramos ter acedido na amostra um grupo de pessoas heterogêneo quanto ao nível etário, uma vez que englobou desde adolescentes até idosos.

Esses dados se assemelham a um estudo realizado sobre o perfil de PDs no Estado de Sergipe, onde, em uma amostra de 128 casos, 121 (94,5\%) tinham idades de até 60 anos, prevalecendo a faixa etária de 21 a 40 anos; apontando que a maior parte dos PDs são adultos jovens em idade economicamente ativa ${ }^{12}$.

Quanto à procedência, notou-se que a maioria dos PDs (56,3\%) era proveniente da região metropolitana do Recife, seguidos daqueles oriundos do interior do Estado de
Pernambuco (43,7\%). Isso mostra que, por ser um hospital de referência em urgência e emergência, atende pacientes procedentes de todo o estado e regiões circunvizinhas; no entanto, há um maior número de mortes por acidentes na metrópole de Recife.

No que se refere ao setor de internação do PD, em que a prevaleceu a emergência, os resultados deste estudo contradizem o que é preconizado na literatura, quando afirma que o cuidado com esse paciente deve ser, preferencialmente, realizado em uma UTI, pois requer uma vigilância constante, por profissionais capacitados no manejo de pacientes críticos $^{13}$.

Ressalta-se que é obrigatória a existência de UTIs em hospitais terciários e secundários com capacidade de 100 leitos, bem como nos especializados que atendam pacientes graves ou de risco. No entanto, apesar de o Brasil ter anunciado o aumento no número de leitos de UTI na última década e mais investimentos, as atuais 16 para quase 17 mil vagas estão muito aquém da demanda ${ }^{14}$.

Sobre o diagnóstico médico, os achados deste estudo diferem da maioria da literatura pesquisada, uma vez que 
mostra como primeira causa o TCE. No entanto, a literatura aponta o AVE como primeira causa, seguido do TCE $8,11-13,15$.

0 percentual expressivo de TCE, neste estudo, expressa que, apesar das campanhas educativas realizadas em todo 0 país sobre a prevenção de acidentes de trânsito, esses eventos ainda respondem como uma das principais causas de mor tes no Brasil.

Em relação à efetividade das doações, os resultados foram divergentes de um estudo realizado no Estado de São Paulo, o qual mostrou que, em uma amostra de 187 PDs, $81,9 \%$ tiveram seus órgãos aproveitados. Por outro lado, o Registro Brasileiro de Transplante aponta que, dos 359 PDs notificados no Estado de Pernambuco no ano de 2011, apenas 67 (18,6\%) tornaram-se doadores com órgãos transplantados, enquanto que, no Brasil, dos 7.238 PDs, 10,6\% tiveram os órgãos captados ${ }^{8,10}$.

Dessa forma, os resultados deste estudo sinalizam que, no Estado de Pernambuco, há um baixo aproveitamento do número de PDs de órgãos e tecidos em relação a outros estados brasileiros, apontando a necessidade para campanhas de educação dos profissionais de saúde e da população em geral.

No tocante às alterações fisiológicas da ME presentes nos PDs, observa-se que os dados alcançados corroboram os da literatura, quando afirmam que a hipotensão arterial surge como a complicação mais frequente da ME, aparecendo em até $81,0 \%$ dos PDs, sendo considerada o agente desencadeador de outras complicações catastróficas, como a parada cardíaca, quando não tratada rápida e adequadamente ${ }^{5}$.

É importante enfatizar que, durante a descarga autonômica, a acentuada liberação de catecolaminas produz grande vasoconstrição, que acarreta em taquicardia, aumento de PA e da demanda de oxigênio do miocárdio, podendo causar isquemia e necrose miocárdica, além de arritmias cardíacas³.

Após cessar a tempestade autonômica, o resultado é a perda do tônus simpático, com profunda vasodilatação e hipotensão arterial grave, sendo esta a alteração fisiológica mais grave da ME, gerando disfunção cardíaca e instabilidade hemodinâmica, que, se não tratadas, devem progredir para assistolia em torno de 72 horas $^{3}$.

Outra alteração muito presente em PDs é a hipotermia, sendo causa muito comum de não realização do exame clínico para abrir o protocolo, uma vez que é capaz de mimetizar a ME devido ao rebaixamento do nível de consciência. É importante ressaltar que é uma das complicações que devem ser tratadas com mais urgência para evitar o retardo nas avaliações do $P D^{16}$.

Esse evento ocorre porque, com a instalação da ME, o hipotálamo deixa de exercer sua função termorreguladora e a consequência é a hipotermia progressiva, com tendência à equalização da temperatura corporal e do ambiente. $\mathrm{A}$ temperatura central em indivíduos normais varia de $36^{\circ} \mathrm{C}$ a $37,5^{\circ} \mathrm{C}$. A vasodilatação extrema, associada à inabilidade de tremer para produzir calor, além da interferência de fatores exógenos, como infusão de grandes volumes de fluidos não aquecidos e a presença de ar condicionado em unidades de emergência ou terapia intensiva, resultam em diminuição da temperatura de forma muito rápida. A hipotermia induz a diversos efeitos deletérios, como disfunção cardíaca, disritmias, coagulopatia e aumento diurese induzido pelo frio $0^{3,6}$.

No tocante à hipernatremia, esta surge como consequência do $\mathrm{DI}$, que ocorre devido ao processo de necrose da neurohipófise e depleção do hormônio antidiurético (ADH). Divergindo dos resultados desta pesquisa, alguns estudos apontam que a hipernatremia ocorre com menor frequência $(54,1 \%)$ do que o DI $(78,0 \%)$, uma vez que a hipernatremia é uma consequência do DI5, 17.

Por outro lado, percebe-se, também, que 0 percentual de pacientes com hiperglicemia $(62,5 \%)$ é inferior ao de DI, mas essa ocorrência pode não ter sido detectada corretamente, uma vez que o controle desse evento deve ser executado através de dosagens seriadas da glicose sanguínea ou através do controle de glicemia capilar, que deve ser realizado, no mínimo, de seis em seis horas. E nem sempre é possível fazer esse procedimento, principalmente em um setor de emergência8.

Em outros estudos, foi encontrada incidência de hiperglicemia diferente da obtida nesta pesquisa, pois, na literatura, cerca de $72,0 \%$ dos doadores apresentam uma glicemia acima de $200 \mathrm{mg} / \mathrm{dL}$ no momento da retirada dos órgãos ${ }^{5,17-18}$.

Quanto à infecção, presente em 25,0\% dos PDs, não é considerada um fator que contraindica o transplante. No entanto, todos os PDs devem ser investigados quanto à presença de agentes infecciosos, e a terapia com antibiótico deve ser indicada nos casos comprovados ou na suspeita de infecção8.

Apesar do baixo índice de úlcera de córnea $(3,1 \%)$ no presente estudo, esta surge como uma complicação da falta de métodos de prevenção, como utilizar gazes umedecidas com solução salina a $0,9 \%$ sobre as pálpebras fechadas ou usar tiras finas de esparadrapo. Comprovando os achados, estudos mostram que sua incidência é geralmente pequena, por isso esse tecido está associado a altos índices de doação e transplante, cuja porcentagem de captações chega a $79,3 \%{ }^{11}$.

Durante o processo de investigação, um fato relevante verificado foi a disponibilidade de alguns profissionais e de todos os familiares dos PDs, por terem permitido que fossem feitas as observações sem quaisquer objeções. No entanto, a pesquisa teve limitações tais como o baixo número de PDs estudados e a qualidade dos registros realizados pela equipe de saúde, em todos os setores pesquisados, principalmente os relacionados às alterações fisiológicas apresentadas pelos PDs e à descrição detalhada dos procedimentos realizados. 


\section{CONCLUSÕES}

As alterações fisiológicas observadas foram: hipotensão arterial (100\%), hipotermia $(75,0 \%)$; hipernatremia $(62,5 \%)$, DI (37,5\%), hiperglicemia (32,3\%), infecção $(25,0 \%)$, hipertensão arterial $(9,4 \%)$ e úlcera de córnea $(3,1 \%)$.

Como visto na literatura, muitos fatores contribuem para a não efetivação do transplante. Dentre eles estão as alterações fisiológicas ocasionadas pela ME. Dessa forma, é importante que esses eventos sejam detectados e tratados, antes que ocorra a parada cardíaca, estimada como uma das principais causas de não efetivação da doação de órgão e primeira causa deste estudo.

0 conhecimento dessas alterações possibilita à equipe de saúde saber como lidar com o PD e prevenir tais consequências para a melhor manutenção dos órgãos e tecidos para transplante.

\section{REFERÊNCIAS}

1.Sociedade Brasileira de Clínica Médica. Programa de Atualização em Medicina de Urgência- PROURGEN. Porto Alegre: Artmed; 2007.

2.Lago P, Piva J, Garcia PC, Troster E, Bousso A, Sarno M0, et al. Brain death: medical management in seven brazilian pediatric intensive care units. J Pediatr. 2007; 83(2): 133-40.

3.Guetti NR, Marques IR. Assistência de enfermagem ao potencial doador de órgãos em morte encefálica. Rev Bras Enferm. 2008; 61(1): 91-7.

4.Padilha KG, Vattimo MFF, Silva SC, Kimura M. Enfermagem em UTI: cuidando do paciente crítico. São Paulo: Manole; 2010.

5.Rech T, Filho EMR. Manuseio do potencial doador de múltiplos órgãos. Rev Bras Ter Intensiva. 2007; 19(2): 197-204.

6.Guimarães HP, Falcão LFR, Orlando JMC. Guia prático de UTI da AMIB. São Paulo: Atheneu; 2009.

7.Associação Brasileira de Transplante de Órgãos -ABTO. Registro Brasileiro de Transplantes. São Paulo; 2011.

8.Domingos GR, Boer LA, Possamai FP. Doação e captação de órgãos de pacientes com morte encefálica. Enferm Bras. 2010; 9(4): 206-12.

9.Westphal GA, Filho MC, Vieira KD, Zaclikevis VR, Bartz MCM, Wanzuita R, et al. Diretrizes para manutenção de múltiplos órgãos no potencial doador falecido. Parte I. Aspectos gerais e suporte hemodinâmico Rev Bras Ter Intensiva. 2011;23(3): 255-268.

10.Lei $n^{0} 10.211$ de 23 de março de 2001. Altera dispositivos da Lei n. 9.434, de 4 de fevereiro de 1997, que dispõe sobre a remoção de órgãos, tecidos e partes do corpo humano parafins de transplante e tratamento. Diário Oficial da República Federativa do Brasil, Brasilia, DF, 23 mar 2001.
11. Moraes EL, Silva LBB, Moraes TC, Paixão NCS, Izumi NMS, Guarino A. 0 perfil de potenciais doadores de órgãos e tecidos. Rev Latino-Am Enfermagem. 2009; 17(5): 716-20.

12.Fusco CC, Marcelino CAG, Araújo MN, Ayoub AC, Martins CP. Perfil dos doadores efetivos de múltiplos órgãos e tecidos viabilizados pela organização de procura de órgãos de uma instituição pública de cardiologia. JBT: j bras transpl 2009; 12: 1109-12.

13. Rodrigues TK, Júnior AA. Estimativa do número de possíveis doadores de órgãos no Estado de Sergipe no ano de 2007. JBT: j bras transpl. 2010; 13: 1329-92.

14. Moraes, EL, Massarollo, MCKB. A recusa familiar para a doação de órgãos e tecidos para transplante. Rev Latino-Am Enfermagem. 2008; 16(3): 458-64.

15.Ministério da Saúde (BR). Coordenação Geral de Atenção Hospitalar DAE/SAS. Credenciamento de leitos de UTI; 2010.

16.Paz ACAC, Ribeiro PCA, Mascarenhas MDM, Silva MV. Caracterização dos doadores de órgãos e tecidos para transplante do estado do Piauí, de 2000 a 2009. Enferm Foco. 2011; 2(2): 124-27.

17.Nogueira EC, Pereira CU. Potencial para obtenção de órgãos em um hospital de urgência de Sergipe. JBT: j bras transpl. 2007; 10: 756-61.

18.Morato EG. Morte encefálica: conceitos essenciais, diagnóstico e atualização. Revista Medica de Minas Gerais. 2009; 19(3): 227- 